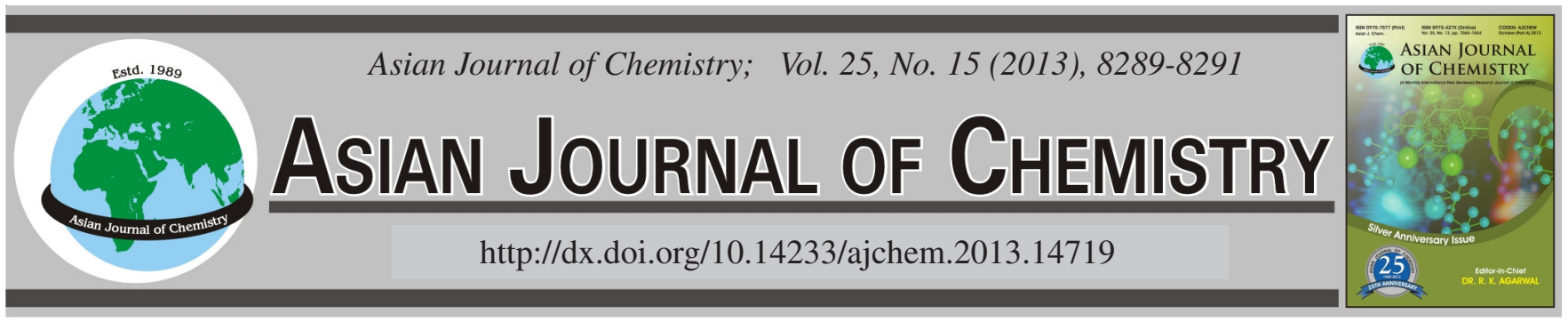

\title{
Sulfonic-Acid-Functionalized Silica Catalyzed, Facile and Efficient Method for the Synthesis of Aryl-14H-dibenzo[a,i]xanthenes
}

\author{
Weilin Li ${ }^{1, *}$, WenLing BiAn ${ }^{2}$ and LiLi Wang ${ }^{2}$
}

${ }^{1}$ School of Pharmacy, Xinxiang Medical University, Xinxiang 453003, Henan Province, P.R. China

${ }^{2}$ Department of Gynecological, The Third Affiliated Hospital to Xinxiang Medical University, Xinxiang 453003, Henan Province, P.R. China

*Corresponding author: Tel./Fax: +86 373 3029879; E-mail: liweilin2002@163.com

(Received: 20 October 2012;

Accepted: 19 August 2013)

AJC-13935

Aryl-14H-dibenzo[a,i]xanthenes were synthesized efficiently by one-pot reaction of $\beta$-naphthol, aldehydes and 2-hydroxy-1,4-naphthoquinone in the presence of sulfonic acid functionalized silica at $110{ }^{\circ} \mathrm{C}$ without solvent. All products were characterized by ${ }^{1} \mathrm{H}$ and ${ }^{13} \mathrm{C}$ NMR, MS techniques and elemental analysis.

Key Words: Dibenzo[a,i]xanthenes, $\beta$-Naphthol, 2-Hydroxy-1,4-naphthoquinone, Sulfonic-acid-functionalized silica, Solvent-free.

ᄂ - - - - - - - - - - - - - - - - - - - - - - - - - - - -

\section{INTRODUCTION}

Xanthenes and particularly benzoxanthenes constitute an important group of oxygen heterocycles which have extended conjugation conferring interesting properties and as have many applications. These compounds possess antiinflammatory ${ }^{1}$, antiviral $^{2}$ and antibacterial activities ${ }^{3}$ and antagonists for the paralyzing acting of zoxazolamine ${ }^{4}$. Furthermore, these compounds can be used as dyes ${ }^{3}, \mathrm{pH}$-sensitive fluorescent materials for visualization of biomolecules ${ }^{5}$ and utilized in laser technologies ${ }^{6}$. Thus, the synthesis of xanthenes derivatives currently is of much importance. Various methods ${ }^{7}$ have been reported for the synthesis of these compounds. Considering the above reports, the development of new and simple synthetic methods for the efficient preparation of dibenzo[a,i]xanthenes is therefore an interesting challenge.

In recent years, the search for environmentally benign chemical processes or methodologies has received much attention $^{8}$. Sulfonic-acid-functionalized silica has been used as an efficient heterogeneous catalyst for many organic transformations because of its low cost, ease of preparation, catalyst recycling and ease of handling. ${ }^{9}$ The catalyst was prepared by the immobilization of propyl thiol on silica using 3-mercaptopropyltrimethoxysilane, followed by the selective oxidation of the thiol groups by aqueous $\mathrm{H}_{2} \mathrm{O}_{2}$ to the sulfonic acid groups (Scheme-I). The catalyst shows high thermal stability (up to $300{ }^{\circ} \mathrm{C}$ ). We now report a simple and efficient route to synthesize aryl-14H-dibenzo [a,i]xanthenes using sulfonic-acidfunctionalized silica as an efficient catalyst under solvent-free conditions (Scheme-II).

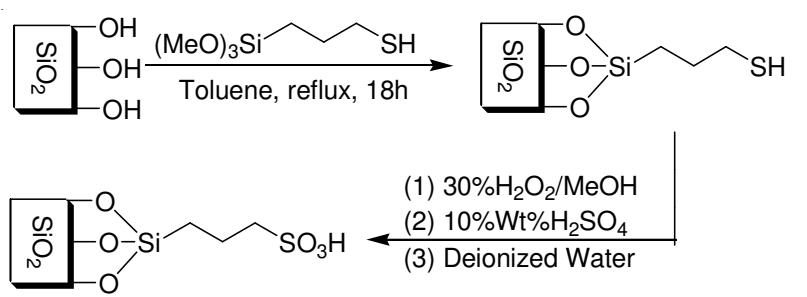

Scheme-I

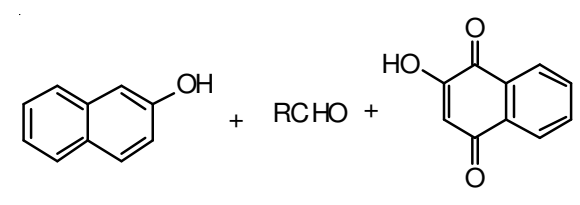

3

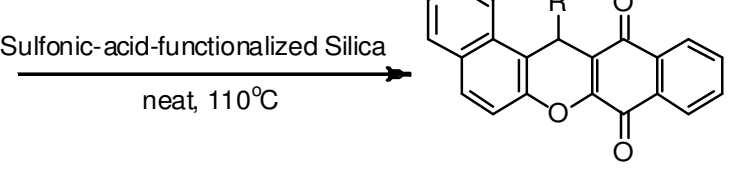

4

Scheme-II

\section{EXPERIMENTAL}

NMR spectra were determined on Bruker AV-400 instrument at room temperature using TMS as internal standard, coupling constants $(J)$ were measured in Hz; IR spectra were determined on FTS-40 infrared spectrometer; Elemental analysis were performed by a Vario-III elemental analyzer. Mass spectra 
were taken on a Macro mass spectrometer (Waters) by electrospray method (ESIMS). Melting points were determined on a XT-4 binocular microscope and were uncorrected; sulfonicacid-functionalized silica was prepared according to literature ${ }^{9}$. Commercially available reagents were used throughout without further purification unless otherwise stated.

General procedure for the preparation of 4: A mixture of $\beta$-naphthol (1 mmol), aldehyde (1 mmol), 2-hydroxy-1,4naphthoquinone $(1 \mathrm{mmol})$ and sulfonic-acid-functionalized silica (200 mg) was heated at $110{ }^{\circ} \mathrm{C}$ for an appropriate time and monitored by thin-layer chromatography (TLC) until the final conversion. After cooling, the reaction mixture was washed with $\mathrm{CHCl}_{3}$ and filtered to recover the catalyst. The solvent was evaporated and the crude product puried by silica gel column chromatography using $\mathrm{CHCl}_{3}$ as eluent to afford the pure product.

14-Phenyl-14H-dibenzo [a,i]xanthene-8,13-dione (4a): Yellow powder, m.p. $319-320^{\circ} \mathrm{C}$; IR $\left(\mathrm{KBr}, \mathrm{v}_{\max }, \mathrm{cm}^{-1}\right)$ : 3081 , 1662, 1630, 1580, 1571, 1373, 1285, 1236, 1213; ${ }^{1} \mathrm{H}$ NMR $\left(\mathrm{CDCl}_{3}, 400 \mathrm{MHz}\right) \delta: 8.15(\mathrm{~d}, 1 \mathrm{H}, J=7.6 \mathrm{~Hz}), 8.11(\mathrm{~d}, 1 \mathrm{H}, J$ $=7.6 \mathrm{~Hz}), 7.96(\mathrm{~d}, 1 \mathrm{H}, J=8.4 \mathrm{~Hz}), 7.91-7.76(\mathrm{~m}, 3 \mathrm{H}), 7.62-$ $7.41(\mathrm{~m}, 6 \mathrm{H}), 7.21(\mathrm{t}, 2 \mathrm{H}, J=15.2 \mathrm{~Hz}), 7.12-7.09(\mathrm{~m}, 1 \mathrm{H})$, $5.95(\mathrm{~s}, 1 \mathrm{H}) ;{ }^{13} \mathrm{C} \mathrm{NMR}\left(\mathrm{CDCl}_{3}, 100 \mathrm{MHz}\right) \delta: 177.33,178.29$, $157.19,147.30,143.12,135.11,131.89,131.22,131.01$, $130.89,130.03,129.51,129.39,128.58,128.55,127.45$, 126.84, 125.53, 124.53, 123.78, 116.88, 116.77, 116.57, 35.16; Anal. calcd. (\%) for $\mathrm{C}_{27} \mathrm{H}_{16} \mathrm{O}_{3}$ : $83.49, \mathrm{H} 4.15$; found (\%): C 83.25, H 4.12.

14-(4-Chlorophenyl)-14H-dibenzo [a,i]xanthene-8,13dione (4b): Yellow powder, m.p. $305-306^{\circ} \mathrm{C}$; IR $\left(\mathrm{KBr}, \mathrm{v}_{\max }\right.$, $\mathrm{cm}^{-1}$ ): 3040, 1665, 1637, 1592, 1577, 1488, 1367, 1286, 1235, 1214; ${ }^{1} \mathrm{H}$ NMR $\left(\mathrm{CDCl}_{3}, 400 \mathrm{MHz}\right) \delta: 8.17(\mathrm{~d}, 1 \mathrm{H}, J=7.6 \mathrm{~Hz})$, $8.12(\mathrm{~d}, 1 \mathrm{H}, J=7.6 \mathrm{~Hz}), 7.93-7.77(\mathrm{~m}, 4 \mathrm{H}), 7.64-7.44(\mathrm{~m}$, $4 \mathrm{H}), 7.35$ (d, 2H, $J=8.4 \mathrm{~Hz}), 7.15(\mathrm{~d}, 2 \mathrm{H}, J=8.4 \mathrm{~Hz}), 5.90$ (s, $1 \mathrm{H}) ;{ }^{13} \mathrm{C} \mathrm{NMR}\left(\mathrm{CDCl}_{3}, 100 \mathrm{MHz}\right) \delta: 176.24,179.18,156.30$, $147.23,141.59,135.17,132.65,131.90,131.39,130.81$, $130.68,130.00,129.96,129.79$, 128.47, 128.70, 128.65, 127.58, 125.67, 124.60, 123.57, 116.79, 116.26, 116.00, 34.62; Anal. calcd. (\%) for $\mathrm{C}_{27} \mathrm{H}_{15} \mathrm{O}_{3} \mathrm{Cl}$ : C 76.69, $\mathrm{H} 3.58$; found (\%): C 76.48, H 3.62.

14-(4-Methoxylphenyl)-14H-dibenzo [a,i]xanthene8,13-dione (4c): Yellow powder, m.p. $279-280^{\circ} \mathrm{C}$; IR ( $\mathrm{KBr}$, $\left.v_{\max }, \mathrm{cm}^{-1}\right): 2917,1663,1633,1591,1575,1367,1286,1249$, 1235, 1212; ${ }^{1} \mathrm{H} \mathrm{NMR}\left(\mathrm{CDCl}_{3}, 400 \mathrm{MHz}\right) \delta: 8.17(\mathrm{~d}, 1 \mathrm{H}, J=$ $8.0 \mathrm{~Hz}), 8.13(\mathrm{~d}, 1 \mathrm{H}, J=7.6 \mathrm{~Hz}), 7.95(\mathrm{~d}, 1 \mathrm{H}, J=8.4 \mathrm{~Hz})$, 7.87-7.76 (m, 4H), 7.62-7.43 (m, 5H), $7.31(\mathrm{~d}, 2 \mathrm{H}, J=8.4$ $\mathrm{Hz}), 5.90$ (s, 1H), 3.69 (s, 3H); ${ }^{13} \mathrm{C} \mathrm{NMR}\left(\mathrm{CDCl}_{3}, 100 \mathrm{MHz}\right)$ $\delta: 178.41,178.37,158.26,157.02,147.23,135.47,135.10$, $131.88,131.17,131.00,130.95,130.00,129.58,129.41$, $129.38,128.53,127.41,125.50,124.50,123.81,117.06$, 116.78, 113.90, 55.13, 34.28; Anal. calcd. (\%) for $\mathrm{C}_{28} \mathrm{H}_{18} \mathrm{O}_{4}$ : C 80.37, H 4.34; found (\%): C 80.50, H 4.27.

14-(4-Methylphenyl)-14H-dibenzo [a,i]xanthene-8,13dione (4d): Yellow powder, m.p. $255-256{ }^{\circ} \mathrm{C}$; IR (KBr, $v_{\max }$, $\mathrm{cm}^{-1}$ ): 2922, 1660, 1637, 1581, 1577, 1364, 1284, 1237, 1213; ${ }^{1} \mathrm{H}$ NMR $\left(\mathrm{CDCl}_{3}, 400 \mathrm{MHz}\right) \delta: 8.15(\mathrm{~d}, 1 \mathrm{H}, J=8.0 \mathrm{~Hz}), 8.11$ $(\mathrm{d}, 1 \mathrm{H}, J=7.6 \mathrm{~Hz}), 7.99(\mathrm{~d}, 1 \mathrm{H}, J=8.0 \mathrm{~Hz}), 7.89-7.76(\mathrm{~m}$, $3 \mathrm{H}), 7.60-7.42(\mathrm{~m}, 4 \mathrm{H}), 7.29(\mathrm{~d}, 2 \mathrm{H}, J=8.0 \mathrm{~Hz}), 7.00(\mathrm{~d}, 2 \mathrm{H}$, $J=7.6 \mathrm{~Hz}), 5.90(\mathrm{~s}, 1 \mathrm{H}), 2.21(\mathrm{~s}, 3 \mathrm{H}) ;{ }^{13} \mathrm{C} \mathrm{NMR}\left(\mathrm{CDCl}_{3}, 100\right.$

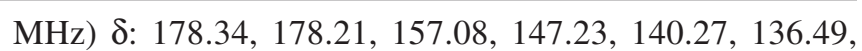
$135.08,131.87,131.16,131.01,130.92,129.99,129.41$, $129.34,129.26,128.52,128.42,127.43,125.50,124.49$, 123.78, 117.03, 116.78, 116.70, 34.70, 20.95; Anal. calcd. (\%) for $\mathrm{C}_{28} \mathrm{H}_{18} \mathrm{O}_{3}$ : $83.57, \mathrm{H} 4.51$; found (\%): C 83.49, $\mathrm{H} 4.63$.

14-(4-Nitrophenyl)-14H-dibenzo[a,i]xanthene-8,13dione (4e): Yellow powder, m.p. 332-333 ${ }^{\circ} \mathrm{C}$; IR ( $\mathrm{KBr}, \mathrm{v}_{\max }$, $\left.\mathrm{cm}^{-1}\right)$ : 3075, 1660, 1638, 1591, 1576, 1519, 1349, 1285, 1236, $1213 ;{ }^{1} \mathrm{H}$ NMR $\left(\mathrm{CDCl}_{3}, 400 \mathrm{MHz}\right) \delta: 8.19(\mathrm{~d}, 1 \mathrm{H}, J=7.6 \mathrm{~Hz})$, $8.16(\mathrm{~d}, 1 \mathrm{H}, J=7.6 \mathrm{~Hz}), 8.07$ (d, 2H, $J=8.8 \mathrm{~Hz}), 7.97-7.81$ $(\mathrm{m}, 4 \mathrm{H}), 7.66-7.49(\mathrm{~m}, 6 \mathrm{H}), 6.06(\mathrm{~s}, 1 \mathrm{H}) ;{ }^{13} \mathrm{C} \mathrm{NMR}\left(\mathrm{CDCl}_{3}\right.$, $100 \mathrm{MHz}) \delta: 178.15,177.98,157.84,150.06,147.29,146.68$, $135.30,131.99,132.73,130.65,130.40,130.38,130.06$, $129.63,129.57,128.83,127.87,125.91,124.77,123.88$, 123.26, 116.83, 115.35, 115.03, 35.25; Anal. calcd. (\%) for $\mathrm{C}_{27} \mathrm{H}_{15} \mathrm{NO}_{5}$ : C 74.81, H 3.49, N 3.23; found (\%): C 74.91, H 3.38, N 3.29.

14-(3- Nitrophenyl)-14H-dibenzo[a,i]xanthene-8,13dione (4f): Yellow powder, m.p. $304-305{ }^{\circ} \mathrm{C}$; IR (KBr, $v_{\max }$, $\mathrm{cm}^{-1}$ ): 3053, 1654, 1635, 1588, 1576, 1528, 1346, 1289, 1239, 1216; ${ }^{1} \mathrm{H} \mathrm{NMR}\left(\mathrm{CDCl}_{3}, 400 \mathrm{MHz}\right) \mathrm{d}: 8.23(\mathrm{~d}, 1 \mathrm{H}, J=8.0 \mathrm{~Hz})$, $8.16(\mathrm{~d}, 1 \mathrm{H}, J=8.0 \mathrm{~Hz}), 8.13(\mathrm{~s}, 1 \mathrm{H}), 8.00-7.82$ (m, 6H), 7.66-7.61 (m, 2H), 7.52-7.41 (m, 3H), $6.06(\mathrm{~s}, 1 \mathrm{H}) ;{ }^{13} \mathrm{C} \mathrm{NMR}$ $\left(\mathrm{CDCl}_{3}, 100 \mathrm{MHz}\right) \delta: 178.24,178.05,157.63,148.56,147.33$, $145.08,135.32,135.08,132.04,131.68,130.60,130.42$, $130.38,130.09,129.59,129.38,128.87,127.82,125.85$, 124.89, 123.32, 123.26, 122.14, 116.97, 115.27, 115.19, 35.20; Anal. calcd. (\%) for $\mathrm{C}_{27} \mathrm{H}_{15} \mathrm{NO}_{5}$ : C 74.82, H3.49, N 3.23; found (\%): C 74.76, H 3.56, N 3.25.

14-(2,4-Dichlorophenyl)-14H-dibenzo[a,i]xanthene8,13-dione (4g): Yellow powder, m.p. $301-302{ }^{\circ} \mathrm{C}$; IR ( $\mathrm{KBr}$, $\left.V_{\max }, \mathrm{cm}^{-1}\right): 3051,1661,1638,1592,1578,1463,1361,1288$, 1238, 1213; ${ }^{1} \mathrm{H} \mathrm{NMR}\left(\mathrm{CDCl}_{3}, 400 \mathrm{MHz}\right) \delta: 8.20(\mathrm{~d}, 1 \mathrm{H}, J=$ 8.4 Hz), 8.15-8.11 (m, 2H), 7.89-7.80 (m, 3H), 7.65-7.45 (m, 4H), 7.31-7.27 (m, 2H), 7.07-7.05 (m, 1H), $6.14(\mathrm{~s}, 1 \mathrm{H}) ;{ }^{13} \mathrm{C}$ $\mathrm{NMR}\left(\mathrm{CDCl}_{3}, 100 \mathrm{MHz}\right) \delta: 178.10,178.05,157.55,147.11$, 139.20, 135.19, 134.01, 133.09, 132.61, 131.79, 131.52, $131.13,130.60,130.13,130.01,129.84,129.47,128.70$, 127.69, 127.49, 125.69, 124.72, 123.71, 116.82, 33.24; Anal. calcd. (\%) for $\mathrm{C}_{27} \mathrm{H}_{14} \mathrm{Cl}_{2} \mathrm{O}_{3}: \mathrm{C} 70.91, \mathrm{H} \mathrm{3.09}$; found (\%): $\mathrm{C}$ $70.82, \mathrm{H} 3.11$.

14-(3,4-Dichlorophenyl)-14H-dibenzo[a,i]xanthene8,13-dione (4h): Yellow powder, m.p. $260-261^{\circ} \mathrm{C}$; IR ( $\mathrm{KBr}$, $\left.V_{\max }, \mathrm{cm}^{-1}\right): 3050,1682,1594,1564,1488,1384,1286,1216$; ${ }^{1} \mathrm{H} \mathrm{NMR}\left(\mathrm{CDCl}_{3}, 400 \mathrm{MHz}\right) \delta: 8.19(\mathrm{~d}, 1 \mathrm{H}, J=7.6 \mathrm{~Hz}), 8.16$ (d, $1 \mathrm{H}, J=7.6 \mathrm{~Hz}), 7.95-7.82(\mathrm{~m}, 4 \mathrm{H}), 7.65-7.43(\mathrm{~m}, 5 \mathrm{H})$, 7.31-7.30 (m, 2H), 5.92 (s, 1H); ${ }^{13} \mathrm{C} \mathrm{NMR}\left(\mathrm{CDCl}_{3}, 100 \mathrm{MHz}\right)$ $\delta: 178.18,178.02,157.55,147.28,143.19,135.24,132.65$, $131.96,131.57,131.05,130.70,130.54,130.42,130.10$, $129.56,128.75,128.15,127.77,125.82,124.73,123.42$, $116.83,115.58,115.44,34.55$; Anal. calcd. (\%) for $\mathrm{C}_{27} \mathrm{H}_{14} \mathrm{O}_{3} \mathrm{Cl}_{2}$ : C 70.91, H 3.09; found (\%): C 70.95, H 3.02.

14-(2-Chlorophenyl)-14H-dibenzo[a,i]xanthene-8,13dione (4i): Yellow powder, m.p. $281-282{ }^{\circ} \mathrm{C}$; IR (KBr, $v_{\max }$, $\mathrm{cm}^{-1}$ ): 3060, 1660, 1638, 1581, 1576, 1360, 1289, 1237, 1213; ${ }^{1} \mathrm{H}$ NMR $\left(\mathrm{CDCl}_{3}, 400 \mathrm{MHz}\right) \delta: 8.24-8.21(\mathrm{~m}, 2 \mathrm{H}), 8.17$ (d, $1 \mathrm{H}, J=7.2 \mathrm{~Hz}), 7.86-7.83(\mathrm{~m}, 3 \mathrm{H}), 7.65-7.29(\mathrm{~m}, 6 \mathrm{H}), 7.08-$ $7.04(\mathrm{~m}, 2 \mathrm{H}), 6.22(\mathrm{~s}, 1 \mathrm{H}) ;{ }^{13} \mathrm{C} \mathrm{NMR}\left(\mathrm{CDCl}_{3}, 100 \mathrm{MHz}\right) \delta$ : $178.20,178.13,157.43,147.12,135.15,133.32,131.76$, 
$131.36,131.30,130.81,130.19,130.13,129.76,129.40$, $128.57,128.23,127.56,127.11,125.57,124.67,123.99$, 116.82, 116.70, 115.61, 33.53; Anal. calcd for $\mathrm{C}_{27} \mathrm{H}_{15} \mathrm{ClO}_{3}: \mathrm{C}$ 76.69, H 3.58; found (\%): C 76.79, H 3.41.

\section{RESULTS AND DISCUSSION}

Initially, we conducted the reaction of $\beta$-naphthol, benzaldehyde and 2-hydroxy-1,4-naphthoquinone in the presence of sulfonic-acid-functionalized silica at different temperatures under solvent-free conditions. The corresponding 14-aryl-14Hdibenzo[a,i]xanthene-8,13-diones was synthesized, The results were summarized in Table- 1 and showed that the reaction using $5 \mathrm{~mol} \%$ sulfonic-acid-functionalized silica at $110^{\circ} \mathrm{C}$ proceeded in highest yield.

TABLE-1

14-ARYL-14H-DIBENZO[a,i]XANTHENE-8,13-DIONES UNDER VARIOUS CONDITIONS ${ }^{\mathrm{a}}$

\begin{tabular}{ccccc}
\hline Entry & $\begin{array}{c}\text { Sulfonic-acid-functionalized } \\
\text { silica }(\mathrm{mg} / \mathrm{mmol})\end{array}$ & $\begin{array}{c}\text { Temp. } \\
\left({ }^{\circ} \mathrm{C}\right)\end{array}$ & $\begin{array}{c}\text { Time } \\
(\mathrm{h})\end{array}$ & $\begin{array}{c}\text { Yield } \\
(\%)^{\mathrm{b}}\end{array}$ \\
\hline 1 & 0 & 100 & 5.00 & 0 \\
2 & 50 & 100 & 2.00 & 59 \\
3 & 100 & 100 & 2.00 & 65 \\
4 & 150 & 100 & 1.00 & 73 \\
5 & 150 & 110 & 0.50 & 82 \\
6 & 200 & 90 & 1.00 & 68 \\
7 & 200 & 100 & 1.00 & 78 \\
8 & 200 & 100 & 0.50 & 83 \\
9 & 200 & 110 & 0.50 & 89 \\
10 & 200 & 120 & 0.50 & 87 \\
11 & 250 & 90 & 0.50 & 80 \\
12 & 250 & 90 & 0.50 & 83 \\
13 & 300 & 90 & 0.25 & 84 \\
\hline
\end{tabular}

${ }^{a}$ Reaction conditions: $\beta$-naphthol ( $\left.1 \mathrm{mmol}\right)$; benzaldehyde ( $\left.1 \mathrm{mmol}\right) ; 2$ hydroxy-1,4-naphthoquinone (1 mmol); neat. ${ }^{b}$ Isolated yield.

Next, to optimize the amount of catalyst and the reaction temperature, the reaction of $\beta$-naphthol, benzaldehyde and 2-hydroxy-1,4-naphthoquinone was studied under solvent-free conditions in the presence of sulfonic-acid-functionalized silica at different temperatures. The results were summarized in Table-1 and showed that the reaction using $200 \mathrm{mg} / \mathrm{mmol}$ sulfonic-acid-functionalized silica at $110{ }^{\circ} \mathrm{C}$ proceeded in highest yield.

In these experiments the catalyst was isolated by filtration and could be recycled up to three times without significant loss of activity. With this optimized procedure in hand, the scope of application of this three-component reaction was examined using different aldehydes as staring materials. As seen from Table-2, the structures of the products were established from their spectral properties (IR, ${ }^{1} \mathrm{H} \mathrm{NMR},{ }^{13} \mathrm{C} \mathrm{NMR}$ and elemental analysis).

The recovered catalyst was reused consecutively 5 times with a minimum of variation of the yields of the products. This reusability demonstrates the high stability. The simplicity, together with the use of an inexpensive, nontoxic and environmentally benign catalyst under solvent-free conditions, is another remarkable feature of the procedure. Sulfonic-acidfunctionalized silica works under heterogeneous conditions, but its reaction centers are highly mobile, as in a homogeneous

\begin{tabular}{|c|c|c|c|c|}
\hline & $\begin{array}{r}\text { PREP } \\
\text { DIBENZC }\end{array}$ & $\begin{array}{r}\text { TABI } \\
\text { ATION O } \\
\text {,I]XANT }\end{array}$ & $\begin{array}{l}2 \\
\text { 4-ARYL- } \\
\text { NE-8,13-I }\end{array}$ & $\mathrm{NES}^{\mathrm{a}}$ \\
\hline Entry & $\mathrm{R}$ & Time (h) & Product & Yield $(\%)^{\mathrm{b}}$ \\
\hline 1 & $\mathrm{C}_{6} \mathrm{H}_{5}$ & 0.75 & $4 a$ & $89(80,54,96)^{c}$ \\
\hline 2 & 4-Cl- $\mathrm{C}_{6} \mathrm{H}_{4}$ & 0.75 & $4 b$ & 87 \\
\hline 3 & 4-MeO- $\mathrm{C}_{6} \mathrm{H}_{4}$ & 0.50 & $4 c$ & 83 \\
\hline 4 & 4-Me- $\mathrm{C}_{6} \mathrm{H}_{4}$ & 0.50 & $4 d$ & 85 \\
\hline 5 & $4-\mathrm{NO}_{2}-\mathrm{C}_{6} \mathrm{H}_{4}$ & 0.50 & $4 e$ & 88 \\
\hline 6 & $3-\mathrm{NO}_{2}-\mathrm{C}_{6} \mathrm{H}_{4}$ & 0.75 & $4 f$ & 86 \\
\hline 7 & $2,4-\mathrm{Cl}_{2}-\mathrm{C}_{6} \mathrm{H}_{3}$ & 1.00 & $4 g$ & 81 \\
\hline 8 & $3,4-\mathrm{Cl}_{2}-\mathrm{C}_{6} \mathrm{H}_{3}$ & 0.75 & $4 h$ & 82 \\
\hline 9 & 2-Cl- $\mathrm{C}_{6} \mathrm{H}_{4}$ & 1.00 & $4 \mathbf{i}$ & 84 \\
\hline
\end{tabular}

${ }^{a}$ Reaction conditions: $\beta$-naphthol $(1 \mathrm{mmol})$; aldehyde $(1 \mathrm{mmol})$; 2 hydroxy-1,4-naphthoquinone ( $1 \mathrm{mmol})$; silica-based sulfonic acid (200 $\mathrm{mg}) ; 110^{\circ} \mathrm{C}$; neat. ${ }^{\mathrm{b}}$ Isolated yield. ${ }^{\mathrm{c}}$ Yield after the fifth cycle.

catalyst. It is an inexpensive and nonhazardous solid acid catalyst. It can easily be handled and removed from the reaction mixture by simple lteration.

\section{Conclusion}

We have developed a novel and highly efficient method for the synthesis of aryl-14H-dibenzo [a,i] xanthene-8,13diones by treatment of aromatic aldehydes, $\beta$-naphthol with 2-hydroxy-1,4-naphthoquinone in the presence of sulfonicacid-functionalized silica as catalyst. The signicant advantages of this methodology are high yields, a simple work-up procedure, cleaner reaction and easy preparation and handling of the catalyst. The catalyst can be recovered by filtration and reused.

\section{ACKNOWLEDGEMENTS}

The authors are pleased to acknowledge the financial support from Xinxiang Medical University.

\section{REFERENCES}

1. J.P. Poupelin, G. Saint-Ruft, O. Foussard-Blanpin, G. Narcisse, G. Uchida-Ernouf and R. Lacroix, Eur. J. Med. Chem., 67, 13 (1978).

2. R.W. Lambert, J.A. Martin, J.H. Merrett, K.E.B. Parkes and G.J. Thomas, PCT Int. Appl. WO 9706178 (1997).

3. B.B. Bhowmik and P. Ganguly, Spectrochim. Acta A, 61, 1997 (2005).

4. R.G. Saint, H.T. Hieu and J.P. Poupelin, Naturwissenschaften, 62, 584 (1975).

5. C.G. Knight and T. Stephens, Biochem. J., 258, 683 (1989).

6. M. Ahmad, T.A. King, B.H. Cha and J. Lee, J. Phys. D: Appl. Phys., 35, 1473 (2002).

7. (a) R.J. Sarma and J.B. Baruah, Dyes Pigments, 64, 91 (2005); (b) A.R. Khosropour, M.M. Khodaei and H. Moghannian, Synlett, 955 (2005); (c) A. Khoramabadizad, S.A. Akbari and A. Shiri, J. Chem. Res., 5, 277 (2005); (d) A.P. Mohamed and P.J. Vaderapura, Bioorg. Med. Chem. Lett., 17, 621 (2007); (e) M.A. Mostafa, S. Mozhdeh and B. Ayoob, Appl. Catal. A, 323, 242 (2007); (f) M.H. Majid, B. Khadijeh, D. Zohreh and F.B. Fatemeh, J. Mol. Catal. A, 273, 99 (2007); (g) R.S. Hamid, G. Majid and H. Asadollah, Dyes Pigments, 76, 564 (2008); (h) S. Mozhdeh, M. Peiman and B. Ayoob, Dyes Pigments, 76, 836 (2008); (i) S. Ko and C.F. Yao, Tetrahedron Lett., 47, 8827 (2006); (j) A.B. Mohammad, M.H. Majid and H.M. Gholam, Catal. Commun., 8, 1595 (2007).

8. D. Choudhary, S. Paul, R. Gupta and J.H. Clark, Green Chem., 8, 479 (2006).

9. Z. Li, X. Ma, J. Liu, X. Feng, G. Tian and A. Zhu, J. Mol. Catal. A, 272, 132 (2007). 